\title{
Effectiveness of Alcohol Brief Intervention in a General Hospital: A Randomized Controlled Trial
}

\author{
JEAN M. MCQUEEN, PH.D., ${ }^{a} *$ TRACEY E. HOWE, PH.D., ${ }^{b}$ CLAIRE BALLINGER, PH.D., ${ }^{c} \&$ JON GODWIN, PH.D. $^{b}$ \\ anstitute of Health and Wellbeing, MRC/CSO Social and Public Health Sciences Unit, University of Glasgow, UK \\ ${ }^{b}$ School of Health \& Life Sciences, Glasgow Caledonian University, UK \\ ${ }^{c}$ National Institute for Health Research Collaboration for Leadership in Applied Health Research and Care, Wessex, University of \\ Southampton, UK
}

\begin{abstract}
Objective: The purpose of this study was to examine the effectiveness of an alcohol brief intervention (ABI) on alcohol consumption in hazardous or harmful drinkers compared with screening alone within a general hospital setting. Method: Following screening, 124 hazardous or harmful drinkers (103 men, ages 18-80 years, score of 3-12 on the Fast Alcohol Screening Test [FAST]) admitted to medical and orthopedic wards during the 13-month recruitment period were randomized to receive an $\mathrm{ABI}$ or control. The intervention group received an $\mathrm{ABI}$ where they were supported to set their own personalized alcohol reduction goals, and both groups received a health information leaflet. Retrospective alcohol consumption for 7 days was reported for the week, before hospital admission and 6 months after it. Results: De-
\end{abstract}

mographics and clinical characteristics at baseline showed no statistical differences between the two groups on all variables except FAST score, which was higher in the intervention group $(p \leq .05)$. A reduction of 85 grams of alcohol per week (95\% CI [162.46, 7.54]) was observed between groups in favor of the intervention group based on changes from baseline. However, there was no significant difference between groups for absolute grams of alcohol per week at 6 months. A significant mean difference in favor of the intervention group $(U=1,537, p=.043)$ was observed for weekly heavy drinking episodes. Conclusions: Our results suggest screening with delivery of $\mathrm{ABI}$ for harmful/hazardous drinkers in a general hospital is beneficial in reducing alcohol consumption compared with screening alone. (J. Stud. Alcohol Drugs, 76, 838-844, 2015)
A ROUND 2 BILLION PEOPLE WORLDWIDE consume alcoholic beverages, and more than 76 million people have alcohol use disorders (Editorial, 2009). Alcohol is a major contributory factor to ill health, chronic disease, and injury globally. In 2012, it was estimated that 3.3 million deaths, or $5.9 \%$ of all deaths, were attributable to alcohol consumption (World Health Organization, 2014). Patterns and volumes of alcohol consumption contribute to many disease categories such as cancer, cardiovascular disease, liver cirrhosis, and injuries to others through the dangerous actions of those intoxicated, such as drink driving and violence (Rehm et al., 2009). The consequences of harmful alcohol use are of major concern to healthcare services. Health professionals working in general hospital environments have regular contact with individuals who misuse alcohol. Historically, interventions were offered only when individuals were diagnosed as alcohol dependent,

Received: December 18, 2014. Revision: July 23, 2015.

The authors thank Professor Carol Tannahill for support and supervision and also NHS Greater Glasgow and Clyde; the College of Occupational Therapists, London; and the Glasgow Centre for Population Health for funding support. Claire Ballinger is supported by the National Institute for Health Research (NIHR) Collaboration for Applied Health Research and Care (CLAHRC) Wessex. The views and opinions expressed are those of the authors and do not necessarily reflect those of the NIHR or NHS.

*Correspondence may be sent to Jean M. McQueen at the MRC/CSO Social and Public Health Sciences Unit, University of Glasgow, G2 3QB; via email at: jeanahp@gmail.com; or on Twitter: @jeanahpm. although evidence has suggested possible benefits from intervening earlier using screening and brief interventions (Nilsen et al., 2008; Wilk et al., 1997).

Alcohol brief interventions (ABIs) consist of a motivational counseling approach involving a time-limited, one-to-one intervention focusing on changing behavior and enhancing an individual's ability to reduce alcohol consumption. ABIs are targeted at non-treatment-seeking, non-alcohol-dependent, hazardous, and harmful drinkers and are intended as an early intervention (Nilsen, 2010). These interventions go beyond advice about reducing alcohol consumption and focus more personally on the individual (McQueen et al., 2006). Previous work has evaluated a range of intercessions categorized as brief interventions, with six key elements widely summarized under the acronym FRAMES (originally described by Bien et al., 1993): feedback, responsibility, advice, menu of strategies, empathy, and selfefficacy. Historically, headlines in the literature highlight a conflicting and confusing picture of brief interventions, for example, "Brief interventions good in theory, but weak in practice" (Roche \& Freeman, 2004), and "Brief interventions have expanded in range, but how they work is still a mystery" (Heather, 2003). There is established evidence relating to ABIs delivered in primary care, with a Cochrane systematic review showing that ABIs reduce the quantity of alcohol consumed by an average of $38 \mathrm{~g}$ per week (Kaner et al., 2007). In contrast, evidence for the delivery of ABIs in a general hospital setting has remained inconclusive and an area requiring further study (Emmen et al., 2004; Saitz, 
2007). The first study into ABI in general hospitals observed improvements in self-reported problems related to alcohol but not alcohol consumption (Chick et al., 1985). A systematic review of $A B I$ in general hospitals included 14 studies with 4,041 participants and identified the need for further research on the optimal content and treatment exposure for ABI (McQueen et al., 2011).

This study examines the effectiveness of screening plus an ABI on alcohol consumption in hazardous or harmful drinkers compared with screening alone within a general hospital setting.

\section{Method}

\section{Study design and baseline data collection}

We used a randomized controlled trial design conducted and reported according to the Consolidated Standards of Reporting Trials (CONSORT) statement (Schulz et al., 2010). Ethical approval was granted by the South Glasgow Research Ethics Committee (ref.: 08/S0710/49). The required sample size was calculated with $95 \%$ confidence and $80 \%$ power to detect a difference between the groups of $75 \mathrm{~g}$ of alcohol per week (nine standard drinks, U.K. units), representing a clinically significant change, with a standard deviation of 200 g. With an estimated 30\% attrition rate, 294 patients were required.

Potentially eligible participants comprised inpatients of any gender, between 18 and 80 years of age. Participants comprised those admitted to the medical and orthopedic wards of a General Hospital in Glasgow, U.K., during the 13-month recruitment period and scoring between 3 and 12 on the 16-point Fast Alcohol Screening Test (FAST) (those scoring $>12$ were deemed more likely to be alcohol dependent) (Hodgson et al., 2002). Those participants identified as cognitively impaired (i.e., scoring less than 23 on the MiniMental State Examination; Folstein et al., 1975), terminally ill, or medically unstable were excluded. Patients already known to the Community Alcohol Team or identified as alcohol dependent were also excluded, as were non-English speakers.

Potentially eligible participants were screened using the FAST by a trained clinical researcher, in collaboration with ward-based nursing and medical staff. The FAST tool consists of four questions designed to measure alcohol misuse and its consequences. Each question is scored between 0 and 4 (maximum score $=16$ ); a score of 3 or more is used to identify alcohol problems. The FAST is quick to administer and score and was devised for use in medical settings where time is a factor (Coulton et al., 2009; Hodgson et al., 2002). Screening took place within the orthopedic and medical wards, 5 days a week, as soon as possible following hospital admission. Eligible patients were given both verbal and written information about the trial, and those willing to participate signed a consent form at face-to-face interviews.

Following consent, baseline data were collected using the FAST tool and the retrospective drinking diary was completed using the 7-day Timeline Followback method (Del Boca \& Darkes, 2003; Sobell \& Sobell, 2003). Each participant was then randomized by an independent therapist not involved in the trial into either the control or ABI (intervention) group via the use of numbered, opaque, sealed envelopes. In this way, the clinical researcher could not influence which participants were allocated to each group, thereby limiting bias through inadequate allocation concealment (Higgins \& Green, 2011).

A different researcher (blind to group allocation) collected data at 6-month follow-up using FAST and the retrospective drinking diary via telephone interviews. Heavy drinking episodes were calculated by counting the number of days per week in which participants consumed $64 \mathrm{~g}$ of alcohol or more (eight U.K. units) for men or $48 \mathrm{~g}$ of alcohol (six U.K. units) for women.

\section{Intervention}

The intervention group received usual hospital care plus screening via the FAST, a health information leaflet, and the ABI. The clinical researcher-a physiotherapist who had received an intensive 2-day training program in $\mathrm{ABI}$ - delivered the intervention and received supervision from the first author (J.M.M.), an occupational therapist with 15 years of clinical and 12 years of supervisory experience. Supervision took place on a weekly basis for the first 2 months, reducing to monthly once the clinical researcher felt confident. The ABI was delivered while participants were still in the hospital; a one-to-one intervention lasting a maximum of 20 minutes. The focus of the ABI was on changing behavior using the FRAMES approach originally described by Bien et al. (1993). During the ABI session, participants were supported using a motivational counseling approach to set their own personalized alcohol-reduction goals. The ABI focused on the following - feedback: using the baseline assessment to support participants to recognize the effect of their drinking, leading to increased responsibility; responsibility: participants are encouraged to think about the role alcohol plays and factors in their lives that may influence their alcohol consumption; advice: specific personalized advice is cocreated by the participant and clinical researcher by asking what might make a difference to reduce alcohol consumption; menu of strategies: participants are encouraged to set their own goals, to suit their current situation and lifestyle; empathy: the intervention is delivered in a nonjudgmental, supportive manner aiming to establish rapport with the participant; and self-efficacy: confidence is built through a personalized plan, considering what success would look like for this individual. 


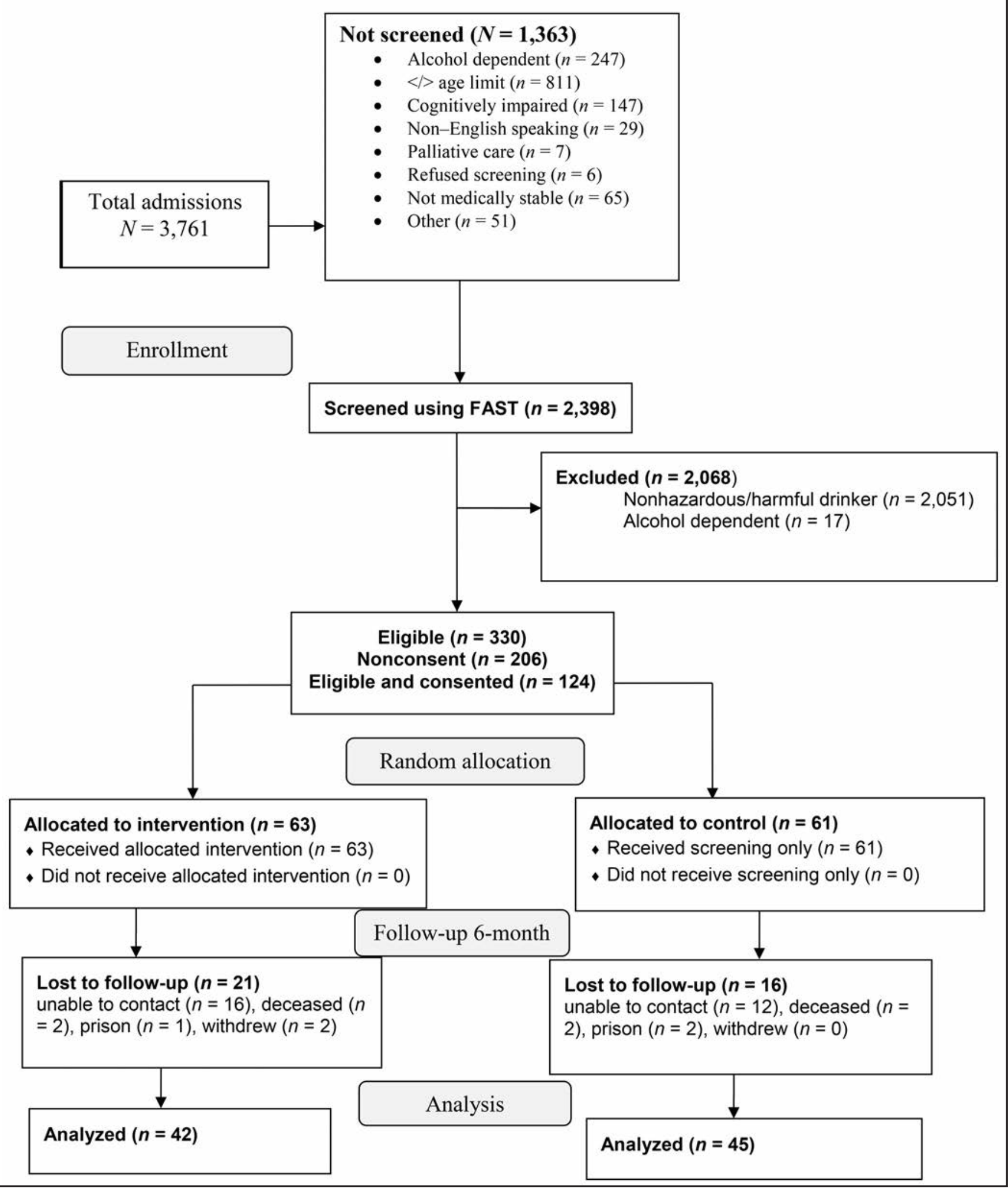

FIGURE 1. Consolidated Standards of Reporting Trials (CONSORT) flow diagram reporting recruitment and retention through trial. FAST $=$ Fast Alcohol Screening Test. 
Because of the potential for hospital stays to be short, ABI was delivered as soon as possible following screening and randomization. Participants in the intervention group also received a personalized follow-up letter or text message from the clinical researcher within 1 month of discharge from the hospital. This discharge contact sought to reinforce the intervention. The precise method of delivery (i.e., letter or text) was based on the participant's stated preference. The control group received usual hospital care plus the same screening (FAST) and general health information leaflet.

\section{Outcomes}

A retrospective drinking diary was used at baseline and 6-month follow-up. Follow-up assessments were completed by telephone by a researcher who was blind to group allocation. The primary outcome measure was changes in grams of alcohol per week from baseline to 6-month follow up. Secondary outcomes were absolute weekly alcohol consumption in grams per week at 6 months follow-up and changes in the number of heavy drinking episodes per week.

\section{Statistical analysis}

The data collected were analyzed using SPSS (SPSS Inc., Chicago, IL). Inferential statistical analyses were used to evaluate the effectiveness of the trial interventions. Primary analyses were by intention to treat. For continuous variables with a nonnormal distribution (grams per week, heavy drinking episodes per week), the Mann-Whitney $U$ test was used to test for statistical significance in consumption between groups.

\section{Results}

\section{Screening and recruitment}

Screening took place over a 13-month period in medical wards and over a 10-month period in orthopedic wards, using the FAST score and inclusion criteria to assess eligibility for entry into the trial (Figure 1). Of the 3,761 patient admissions, 2,398 patients were screened; 330 (14\%) had a FAST score of 3-12 points (indicative of hazardous/harmful drinking) and were therefore eligible for inclusion in the trial. Of these 330 patients, 124 (38\%) agreed to participate, provided consent, and were randomized to one or another arm of the trial: 63 to the intervention group and 61 to the control group.

Although the final recruitment numbers are lower from the orthopedic wards $(n=49)$ than from the medical wards $(n=75)$ (partly because of the shorter period of recruitment and the lower number of patients admitted to the orthopedic wards), proportionately more hazardous/harmful drinkers (with a FAST score of 3-12 points) were identified in the orthopedic wards $(22 \%, n=136)$ compared with the medical wards $(11 \%, n=194)$.

\section{Baseline characteristics}

Baseline characteristics of the participants are shown, by intervention status, in Table 1. The intervention and control groups were analogous for most baseline characteristics (e.g., age, gender, employment status, and units/grams of alcohol per week). The only difference was seen in relation to the mean FAST score. This was higher in the intervention group, which had a mean score of 6 (range: 3-12) compared with the control group, which had a mean score of 5 (range: 3-12). Based on the Mann-Whitney $U$ test, this represented a statistically significant difference, with a mean difference of $1.2(U=1,343, p=.003)$.

\section{Follow-up assessment}

Eighty-seven participants were successfully followed up at 6 months, a 70\% follow-up rate. More participants were lost to follow-up in the intervention group $(n=21)$ than in the control group $(n=16)$, but this difference was not statistically significant, $\chi^{2}(1)=0.78, p=.436$. Further analysis examined whether characteristics of participants lost to follow-up were different from those who completed the trial. Those participants lost to follow-up tended to be younger than those who continued in the trial, with mean ages of 40 and 47 years, respectively $(p=.03$ ), based on results of independent sample $t$ tests.

\section{Effects of the intervention}

Primary outcome measure. The primary outcome measure was changes from baseline in grams of alcohol per week. Participants in the intervention group reduced their alcohol consumption more than those in the control group. The average decrease for the intervention group was 97 g per week $(n=63)$ compared with $12 \mathrm{~g}$ per week for the control group $(n=61)$. The difference between these means was $85 \mathrm{~g}$ reduction per week $(95 \%$ CI [162.46, 7.54]) in favor of the intervention group $(p=.03)$. This represents both a statistically significant and clinically important difference (Table 2).

Secondary outcome measures. Absolute alcohol consumption in grams per week at 6 months revealed that those participants in the intervention group were drinking on average $169 \mathrm{~g}$ of alcohol per week (21.1 U.K. units) compared with $219 \mathrm{~g}$ of alcohol per week (27.4 U.K. units) in the control group. Although those participants in the intervention group were drinking less than those in the control group by more than 6 U.K. units $(50 \mathrm{~g})$ per week, this difference was not statistically significant $(U=1,727, p=.33)$ (Table 2$)$. 
TABLE 1. Demographic and clinical characteristics of participants at baseline $(n=124)$

\begin{tabular}{|c|c|c|c|c|}
\hline Variable & $\begin{array}{c}\text { Total } \\
\text { sample } \\
(n=124)\end{array}$ & $\begin{array}{c}\text { Intervention } \\
\text { group } \\
(n=63)\end{array}$ & $\begin{array}{c}\text { Control } \\
\text { group } \\
(n=61)\end{array}$ & $p$ \\
\hline Age, in years & & & & $.546^{a}$ \\
\hline$M(S D)$ & 45 (17) & $46(16)$ & $43(17)$ & \\
\hline Range & $18-80$ & $18-80$ & $18-79$ & \\
\hline FAST & & & & $.003^{*, b}$ \\
\hline$M(S D)$ & $5(2.4)$ & $6(2.4)$ & $5(2.3)$ & \\
\hline Range & $3-12$ & $3-12$ & $3-12$ & \\
\hline Units per week & & & & $.229^{b}$ \\
\hline$M(S D)$ & $32(30)$ & $35(31)$ & $28(30)$ & \\
\hline Range & $0-194^{c}$ & $0-162^{c}$ & $0-194^{c}$ & \\
\hline Grams per week & & & & $.241^{b}$ \\
\hline$M(S D)$ & $246(243)$ & $270(177)$ & $220(154)$ & \\
\hline Range & $0-1,550^{c}$ & $0-1,292^{c}$ & $0-1,550^{c}$ & \\
\hline $\begin{array}{l}\text { Heavy drinking episodes } \\
\text { per week }\end{array}$ & & & & $.207^{b}$ \\
\hline$M(S D)$ & $1.6(1.5)$ & $1.78(1.6)$ & $1.41(1.3)$ & \\
\hline Range & $0-6$ & $0-6$ & $0-6$ & \\
\hline Alcohol-free days per week & & & & $.957^{a}$ \\
\hline$M(S D)$ & $4.8(1.8)$ & $4.8(1.7)$ & $4.8(1.8)$ & \\
\hline Range & $0-7$ & $1-7$ & $0-7$ & \\
\hline Gender, $n(\%)$ & & & & $.553^{d}$ \\
\hline Male & $103(83 \%)$ & $52(83 \%)$ & $51(84 \%)$ & \\
\hline Living status, $n(\%)$ & & & & $.108^{d}$ \\
\hline Alone & $33(27)$ & $19(30 \%)$ & $14(23 \%)$ & \\
\hline Family/others & $66(53)$ & $36(57 \%)$ & $30(48 \%)$ & \\
\hline Unknown & $25(20)$ & $8(13 \%)$ & $17(27 \%)$ & \\
\hline Employment status, $n(\%)$ & & & & $.899^{d}$ \\
\hline Full time & $65(53 \%)$ & $32(51 \%)$ & $33(54 \%)$ & \\
\hline Part time & $4(3 \%)$ & $2(3 \%)$ & $1(1 \%)$ & \\
\hline Student & $3(2 \%)$ & $2(3 \%)$ & $2(3 \%)$ & \\
\hline Unemployed & $24(19 \%)$ & $12(19 \%)$ & $12(20 \%)$ & \\
\hline Retired & $22(18 \%)$ & $13(21 \%)$ & $9(15 \%)$ & \\
\hline Not recorded & $6(5 \%)$ & $2(3 \%)$ & $4(7 \%)$ & \\
\hline Ward, $n(\%)$ & & & & $.199^{d}$ \\
\hline Medical & $75(60 \%)$ & $42(67 \%)$ & $33(54 \%)$ & \\
\hline Orthopedic & $49(40 \%)$ & $21(33 \%)$ & $28(46 \%)$ & \\
\hline
\end{tabular}

${ }^{a}$ Independent $t$ sample; ${ }^{b}$ Mann-Whitney $U$; ${ }^{c}$ some participants did not drink in the week before admission but still scored FAST positive (i.e., $\geq 3$ ); those who identified that this was atypical of their usual drinking pattern were included in the study $(n=16) ;{ }^{d}$ chi-square. $* p<.05$.

A heavy drinking episode equates to $64 \mathrm{~g}$ of alcohol or more for men or $48 \mathrm{~g}$ of alcohol or more for women on one occasion (Institute of Alcohol Studies, 2013). Those participants in the control group had no change in the mean number of heavy drinking episodes per week, whereas those in the intervention group showed a decrease of 0.65 days (or just over half a day), representing a mean difference of -0.65 episodes $(U=1,537, p=.043)$ (Table 2).

\section{Discussion}

Following screening with the FAST, ABI delivered in addition to usual care within a general hospital setting to harmful and hazardous drinkers was significantly more effective in reducing alcohol consumption (mean difference of $85 \mathrm{~g}$ per week) than just screening and routine hospital care at 6 months (primary outcome). However, although there was a

TABLE 2. Summary of outcomes at 6-month follow-up comparison between control and intervention group ( $n$ $=124)$

\begin{tabular}{|c|c|c|c|c|c|}
\hline Outcome & Variable & $\begin{array}{l}\text { Total sample } \\
\quad(n=124)\end{array}$ & $\begin{array}{c}\text { Intervention } \\
\text { group }(n=63)\end{array}$ & $\begin{array}{c}\text { Control } \\
\text { group }(n=61)\end{array}$ & $p$ \\
\hline Changes from baseline, & $M(S D)$ & $-50(223)$ & $-97(228)$ & $-12(212)$ & $.03^{a}$ \\
\hline grams of alcohol per week & Range & & $-1,080-392$ & $-643-905$ & \\
\hline Absolute weekly alcohol & $M(S D)$ & $194(226)$ & $169(184)$ & $219(263)$ & $.33^{a}$ \\
\hline Consumption, grams per week & Range & $0-1,549$ & $0-864$ & $0-1,549$ & \\
\hline Changes in heavy drinking & $M(S D)$ & $-0.33(1.72)$ & $-0.65(1.75)$ & $0(1.64)$ & $.043^{a}$ \\
\hline episodes, no. of days & Range & $-6-6$ & $-6-6$ & $-5-6$ & \\
\hline
\end{tabular}

Notes: No. $=$ number. ${ }^{a}$ Mann-Whitney $U$. 
trend toward a greater reduction in the ABI group, there was no significant difference between the groups for absolute alcohol consumption. In addition to these clinically important gains, participants in the ABI group were also more likely to report a reduction in the number of heavy drinking episodes at 6-month follow-up (secondary outcome). If this small weekly change was consistent, then over a 6-month period this would equate to approximately 16 heavy drinking episodes per person in the $\mathrm{ABI}$ group.

The variation in results between primary and secondary outcomes may be linked to the method of analysis. The primary outcome - changes from baseline in grams of alcohol per week-is calculated using baseline grams of alcohol per week minus grams of alcohol per week at 6-month follow-up. This type of analysis is less vulnerable to variability in participants' drinking and, therefore, can be more efficient and powerful than simply comparing final values. However, the statistical significance of the treatment effect by the two methods will depend on the degree of correlation between the results. If correlation is high, as in this trial, using only the follow-up score, mean alcohol consumption in grams per week will lose information and the change score is more likely to be significant; however, if correlation is low, use of the change score will add variation and the follow-up score is more likely to be significant (Vickers \& Altman, 2001). Using both outcomes in one study has advantages in terms of triangulation but only if the different measures point to results in the same direction. In this study, despite the differences in statistical significance, both outcomes point to the intervention group consuming less alcohol at follow-up.

These results concur with those presented in a systematic review of $\mathrm{ABI}$ in a general hospital setting, confirming the effectiveness of ABI in reducing alcohol consumption (McQueen et al., 2011). The systematic review of 14 studies indicates that patients receiving brief interventions have a greater reduction in alcohol consumption compared with those in control groups at 6- and 9-month follow-ups. However, the small number of studies, relatively small sample sizes, and heterogeneity between the studies resulted in large confidence intervals and less certainty as to the true effects of ABI. This systematic review identified the need for more studies within general hospital settings (McQueen et al., 2011). Synergies between the findings are reassuring, and future updates of the systematic reviewincluding newly published studies such as this one-will add to the evidence base. Another systematic review examining the effectiveness of $\mathrm{ABI}$ in primary care indicated that based on 22 studies, brief intervention resulted in significant reductions in weekly alcohol consumption for men (Kaner at al., 2007).

The present study has several strengths. Of 3,761 admissions during the 13-month recruitment period, 2,398 patients were assessed for eligibility by screening with FAST, and
330 patients were eligible for recruitment. The randomization process - administered by an independent therapist using sequentially numbered, opaque, sealed envelopesensured that participants had an equal chance of being allocated to either the intervention or control group. The use of assessors blind to group allocation further minimized the risk of bias. A sample size calculation was used to determine recruitment targets based on an estimated $30 \%$ attrition rate. The attrition rate was correct because $70 \%$ of the participants were followed up 6 months later. This compares with retention rates of between $68 \%$ and $100 \%$ in the 14 studies included in the systematic review of ABI in general hospital settings (McQueen et al., 2011). A further strength of this study is that an intention-to-treat analysis was used.

The present study has limitations. The sample size achieved for the trial of 87 completers was smaller than the required sample size calculation of 294 (including a 30\% attrition rate); thus, power was not achieved. The potential reasons for not achieving the target recruitment may have been due to several factors. The sample was specifically selected to include nondependent drinkers; therefore, the results may not apply to dependent drinkers. We were not able to screen all admissions, and recruitment took place only 5 days a week, with no cover for annual leave. This was compounded by the rapid nature of admission and discharge for some patients.

Issues with consent, however, may clearly have a bearing on overall findings. Of those patients who were eligible, there was only endpoint data for 87 of 330 patients, only $26 \%$ of those who were actually eligible for the intervention. Those participants who did not consent cited reasons such as the fear of being stigmatized, concern their welfare benefits may be affected, or that the individual may not have perceived his or her alcohol consumption to be problematic. Offering participants incentives could potentially have improved recruitment; however, there is considerable sensitivity and some controversy over the use of incentives in research, with much debate about whether such practice is ethical (Grant \& Sugarman, 2004).

The only difference between the groups at baseline was that the mean FAST score was higher in the intervention group ( $M=6$, range: $3-12)$, compared with the control group $(M=5$, range: $3-12)$. However, participants still were in the harmful/hazardous range.

Telephone interviews were used for convenience at 6-month follow-up to collect data, and it is acknowledged that the loss of nonverbal body language may be a limitation in this study. The absence of visual cues via telephone could result in the loss of contextual and nonverbal data, compromising rapport, probing, and interpretation of responses. Yet, it has been suggested that telephone interviews may allow respondents to feel relaxed and able to disclose sensitive information, and evidence is lacking that they produce lower quality data (Novick, 2008). 


\section{Conclusions}

Results from this study suggest that there are important clinical benefits to delivering ABI to hazardous/harmful drinkers in general hospitals, with a significantly greater reduction in alcohol consumption among those in the intervention group at 6-month follow-up. In addition, ABI is more effective at achieving a reduction in reported episodes of heavy drinking than screening and routine hospital care. These results suggest that there are benefits to increasing the provision of this type of intervention with this population in general hospitals. If implemented in most general hospitals, this could result in a clinically important reduction in hazardous or harmful levels of alcohol consumption. However, it is unclear if these benefits are sustained in the longer term (i.e., beyond the 6-month follow-up period). Potentially, there is the risk that alcohol consumption may rebound to baseline, and further research with additional follow-up and/or the provision of booster sessions could be worthy of investigation.

\section{References}

Bien, T. H., Miller, W. R., \& Tonigan, J. S. (1993). Brief interventions for alcohol problems: A review. Addiction, 88, 315-336. doi:10.1111/j.1360-0443.1993.tb00820.x.

Chick, J., Lloyd, G., \& Crombie, E. (1985). Counselling problem drinkers in medical wards: A controlled study. British Medical Journal (Clinical Research Edition), 290, 965-967. doi:10.1136/bmj.290.6473.965.

Coulton, S., Perryman, K., Bland, M., Cassidy, P., Crawford, M., Deluca, P., . . . Shepherd, J. (2009). Screening and brief interventions for hazardous alcohol use in accident and emergency departments: A randomised controlled trial protocol. BMC Health Services Research, 9, 114. doi:10.1186/1472-6963-9-114

Del Boca, F. K., \& Darkes, J. (2003). The validity of self-reports of alcohol consumption: State of the science and challenges for research. Addiction, 98, Supplement 2, 1-12. doi:10.1046/j.1359-6357.2003.00586.x.

Editorial: Alcohol misuse needs a global response [Editorial]. (2009). The Lancet, 373, 433. doi:10.1016/S0140-6736(09)60146-X.

Emmen, M. J., Schippers, G. M., Bleijenberg, G., \& Wollersheim, H. (2004). Effectiveness of opportunistic brief interventions for problem drinking in a general hospital setting: Systematic review. BMJ, 328, 318. doi:10.1136/bmj.37956.562130.EE.

Folstein, M. F., Folstein, S. E., \& McHugh, P. R. (1975). "Mini-mental state": A practical method for grading the cognitive state of patients for the clinician. Journal of Psychiatric Research, 12, 189-198. doi:10.1016/0022-3956(75)90026-6.

Grant, R. W., \& Sugarman, J. (2004). Ethics in human subjects research: Do incentives matter? Journal of Medicine and Philosophy, 29, 717-738. doi:10.1080/03605310490883046.

Heather, N. (2003). Brief alcohol interventions have expanded in range but how they work is still mysterious. Addiction, 98, 1025-1026. doi:10.1046/j.1360-0443.2003.00441.x.

Higgins, J. P. T., \& Green, S. (Eds.). (2011). Cochrane Handbook for Sys- tematic Reviews of Interventions Version 5.1.0 (updated March 2011). The Cochrane Collaboration. Retrieved from www.cochrane-handbook. org

Hodgson, R., Alwyn, T., John, B., Smith, A., Newcombe, R., Morgan, C., \& Waller, S. (2002). Manual for the Fast Alcohol Screening Test (FAST): Fast screening for alcohol problems. London, England: Health Development Agency and University of Wales College of Medicine. Retrieved from http://alcoholresearchuk.org/downloads/finalReports/ AERC_FinalReport_0005.pdf

Institute of Alcohol Studies. (2013). A good measure: Units and drinking guidelines. Retrieved from http://www.ias.org.uk/Alcohol-knowledgecentre/Consumption/Factsheets/A-good-measure-Units-and-drinkingguidelines.aspx

Kaner, E. F., Dickinson, H. O., Beyer, F. R., Campbell, F., Schlesinger, C., Heather, N., . . Pienaar, E. D. (2007). Effectiveness of brief alcohol interventions in primary care populations. Cochrane Database of Systematic Reviews, Issue 2, Art. No.: CD004148. doi:10.1002/14651858. CD004148.pub3.

McQueen, J., Allan, L., \& Mains, D. (2006). Brief motivational counselling for alcohol abusers admitted to medical wards. British Journal of Occupational Therapy, 69, 327-333. doi:10.1177/030802260606900705.

McQueen, J., Howe, T. E., Allan L., Mains, D., \& Hardy, V. (2011). Brief interventions for heavy alcohol users admitted to general hospital wards. Cochrane Database of Systematic Reviews, Issue 8. Art. No.: CD005191. doi:10.1002/14651858.CD005191.pub3.

Nilsen, P. (2010). Brief alcohol intervention - where to from here? Challenges remain for research and practice. Addiction, 105, 954-959. doi:10.1111/j.1360-0443.2009.02779.x.

Nilsen, P., Kaner, E., \& Babor, T. F. (2008). Brief intervention, three decades on. An overview of research findings and strategies for more widespread implementation. Nordic Studies on Alcohol and Drugs, 25, 453-467.

Novick, G. (2008). Is there a bias against telephone interviews in qualitative research? Research in Nursing \& Health, 31, 391-398. doi:10.1002/ nur.20259.

Rehm, J., Mathers, C., Popova, S., Thavorncharoensap, M., Teerawattananon, Y., \& Patra, J. (2009). Global burden of disease and injury and economic cost attributable to alcohol use and alcohol-use disorders. The Lancet, 373, 2223-2233. doi:10.1016/S0140-6736(09)60746-7.

Roche, A. M., \& Freeman, T. (2004). Brief interventions: Good in theory but weak in practice. Drug and Alcohol Review, 23, 11-18. doi:10.108 0/09595230410001645510.

Saitz, R. (2007). Screening and brief intervention enter their 5th decade. Substance Abuse, 28, 3-6. doi:10.1300/J465v28n03_02.

Schulz, K. F., Altman, D. G., \& Moher, D., \& the CONSORT Group. (2010). CONSORT 2010 statement: Updated guidelines for reporting parallel group randomised trials. BMJ, 340, c332. doi:10.1136/bmj.c332.

Sobell, L. C., \& Sobell, M. S. (2003). Alcohol consumption measures. In J. P. Allan \& V. B. Wilson (Eds.), Assessing alcohol problems: A guide for clinicians and researchers. Bethesda, MD: National Institute on Alcohol Abuse and Alcoholism.

Vickers, A. J., \& Altman, D. G. (2001). Statistics notes: Analysing controlled trials with baseline and follow up measures. BMJ, 323, 11231124. doi:10.1136/bmj.323.7321.1123.

Wilk, A. I., Jensen, N. M., \& Havighurst, T. C. (1997). Meta-analysis of randomized control trials addressing brief interventions in heavy alcohol drinkers. Journal of General Internal Medicine, 12, 274-283. doi:10.1007/s11606-006-5063-z.

World Health Organization. (2014). Global status report on alcohol and health. Geneva. Switzerland: Author. 